\title{
A MECHANICALLY-BALANCED, DRIE ROTARY ACTUATOR FOR A HIGH-POWER TUNABLE LASER
}

\author{
Hal Jerman and John D. Grade \\ iolon, Inc. \\ 1870 Lundy Ave, San Jose, CA 95131
}

\begin{abstract}
A DRIE actuator has been developed to allow tuning of a 20$\mathrm{mW}$ output power, external-cavity laser operating over the entire C- or L-band for long-haul telecommunications applications. This actuator has the ability to rotate a large, externally-fabricated mirror about a point in space selected to achieve the desired continuous tuning range. The actuator has been designed to be mechanically balanced and therefore relatively immune to accelerations in the plane of the device.
\end{abstract}

\section{APPLICATION BACKGROUND}

Long-haul telecommunications systems have traditionally used fixed wavelength lasers to transmit data over fiber optic links. Systems currently in operation can multiplex light from as many as 100 fixed wavelength lasers, each modulated at up to $10 \mathrm{~Gb} / \mathrm{s}$, at wavelengths around $1550 \mathrm{~nm}$ on a single optical fiber. Optical channels have been assigned by the International Telecommunications Union (ITU) at regular intervals, which in current systems are at $100 \mathrm{GHz}(0.8 \mathrm{~nm})$ or $50 \mathrm{GHz}$ spacing, although the trend is to move to $25 \mathrm{GHz}$ channel spacing in the near future. There is an inventory problem associated with supplying so many different fixed wavelength devices and current systems employing fixed wavelength lasers cannot be rapidly configured to provision new optical links in the network. Tunable lasers solve these problems if they can provide optical performance equal to or better than the current fixed wavelength lasers at an acceptable price.

\section{OPTICAL DESIGN}

The optical design of this laser is a modification of the classic Littman-Metcalf arrangement, Fig. 1, where a Fabry-Perot laser diode chip includes one AR-coated facet [1]. Light from that facet is collimated and strikes a diffraction grating at grazing incidence. Light of different wavelengths is diffracted at different angles, thus the diffraction grating serves as an optical filter in the cavity. A beam at the angle associated with the desired output wavelength is retro-reflected by a mirror mounted on a microactuator, sending the light back to the diode thus supporting lasing at that wavelength in the cavity. Changing the angle of the retroreflecting mirror varies the wavelength of the laser light.

The phase of the light in the cavity must also be controlled to eliminate mode hops, or abrupt changes in wavelength of light, as the laser is tuned. The cavity length and mirror angle must be simultaneously changed so that there are always an equal integer number of waves in the cavity. The geometrical arrangement to achieve this was originally described by Metcalf and Liu [2], where the tuning mirror rotates about a proscribed point in space. In traditional tunable lasers of this design, rotation is controlled by having a mechanical bearing at that location, although it is difficult to insure that the mechanical pivot is in the correct location for a given set of optical components.
Alternative designs for external cavity lasers including micromechanical elements for tuning have included vertical-cavity, surface-emitting lasers (VCSEL's) $[\mathbf{3 , 4}]$ and Fabry-Perot lasers including a moving rear-facet reflector [5], but these designs have not yet achieved the combination of power, tuning range, and optical performance needed for long-haul telecommunications applications.

In our design, a flexural suspension has been designed to place a virtual pivot point for a rotating mirror remote from the actuator device itself, to achieve the required phase control [6]. A pivot set by a flexural suspension is inherently free from friction and hysteresis effects common in mechanical bearings and tends to be reproducible and easy to adjust with a closed-loop control system.

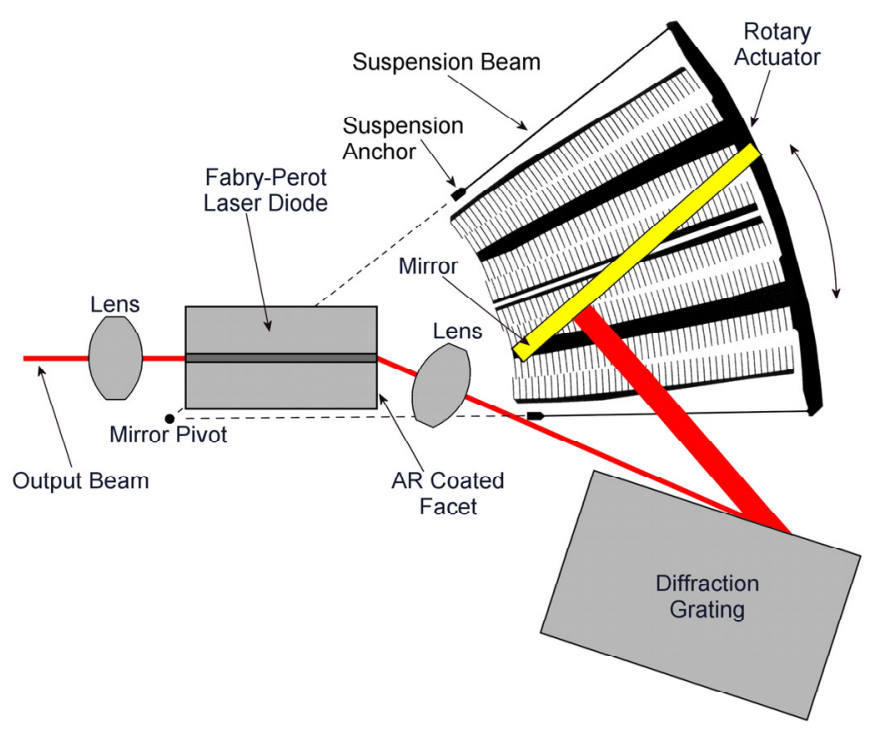

Figure 1. Schematic view of a modified Littman-Metcalf external cavity laser. Laser is tuned by rotating a mirror mounted on a MEMS actuator about a virtual pivot point selected to maintain the correct phase relationship in the cavity.

A separately fabricated mirror with a high-reflectivity gold coating is attached to mounting pads on the actuator and extends $600 \mu \mathrm{m}$ out-of-plane to intercept the light. The range of motion of the mirror required to tune over a given range of wavelengths is set by the line spacing of the grating and the operating wavelength. In this case, with $1200 \mathrm{line} / \mathrm{mm}$ grating period, the wavelength changes about $7.5 \mathrm{~nm}$ for each degree of rotation around the mirror virtual pivot. Thus to tune over a $38 \mathrm{~nm}$ wavelength range, the mirror must rotate a total of almost 5 degrees, and the distal end of the mirror must travel over $300 \mu \mathrm{m}$ during full range tuning.

The individual optical elements are mounted on a temperature-controlled ceramic substrate. Controlling the 
temperature to about $25^{\circ} \mathrm{C}$ improves the output power of the diode, increases diode life, and provides a stable cavity geometry.

In addition, an etalon wavelength locker assembly is included which is needed both to insure that the laser is accurately tuned to the desired ITU channel, and also to allow rapid closed-loop servo tuning of the laser. These lockers use the periodic transmission spectrum of a Fabry-Perot etalon, where the etalon thickness and angle are adjusted so that a specified transmission intensity (e.g. $50 \%$ ) corresponds to sequential ITU channel wavelengths.

By including this locker on the same temperature-controlled platform as the laser, the stability of the etalon used to set the channel wavelength is improved. The frequency can be held to an absolute accuracy of about $+/-0.7 \mathrm{GHz}$ at a channel frequency of about $195 \mathrm{THz}$ over temperature and the lifetime of the device, which represents a maximum frequency error of about $+/-3.5 \mathrm{ppm}$. The laser must be controlled to a resolution of about $0.1 \mathrm{GHz}$ in order to meet this specification, or about 1 part in 50,000 of the full-scale range of the actuator. The laser and locker are hermetically sealed in a conventional hybrid butterfly package. A schematic view of the package configuration is shown in Fig. 2.

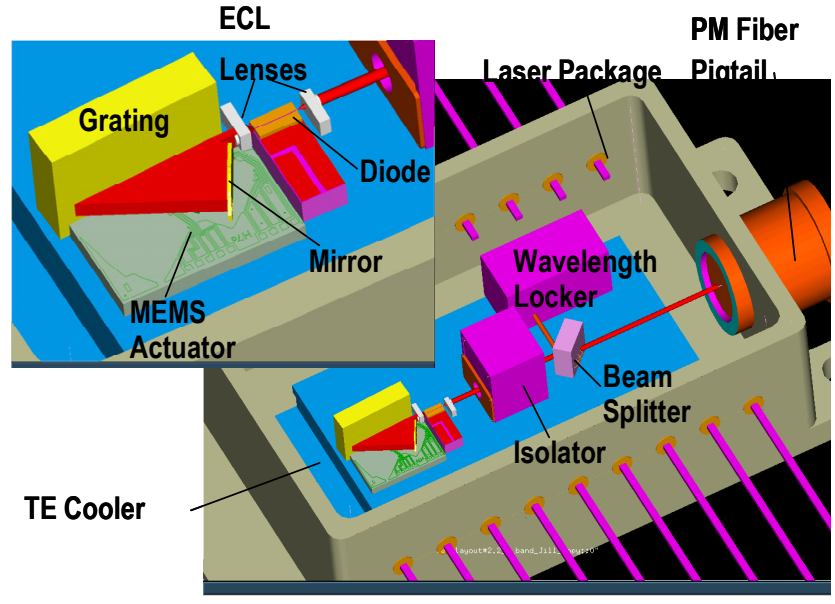

Figure 2. Schematic view of the packaged external cavity laser including the optical isolator, wavelength locker section, and fiber pigtail assembly. The 19x26 $\mathrm{mm}$ butterfly package is hermetically sealed.

\section{ACTUATOR DESIGN}

The fabrication of this actuator is identical to that used for an optical switch described at HH2000 [7]. A cavity is formed in an oxidized substrate, whose extent will define which parts of the structure are fixed to the substrate and which are free to move. A second wafer is fusion-bonded to this etched surface and ground and polished to a thickness of $85 \mu \mathrm{m}$. After contact holes and aluminum bonding pads are defined, the $85-\mu \mathrm{m}$ thick Si layer is DRIE etched to form the suspended electrostatic actuator, substrate attachment areas, bonding pads, and electrical interconnect. With currently available etching tools, $4-\mu \mathrm{m}$ wide suspension elements and $10-\mu \mathrm{m}$ comb gaps are reliably obtained. A plan view of one actuator design is shown in Fig. 3, showing the actuator in relation to the laser diode chip, collimating lens, and a portion of the diffraction grating.

This and similar actuators have been designed to use separately fabricated optical elements, such as mirrors, thin-film optical filters, lenses, and diffraction gratings. This results in a relatively simple actuator process, with correspondingly high yield, and optical elements that can be fabricated in a variety of material systems with no concern about how their fabrication process would need to be integrated with the actuator.

This actuator requires $150 \mathrm{~V}$ to rotate a $600-\mu \mathrm{m}$ tall $\times 1.7-\mathrm{mm}$ long mirror through $5^{\circ}$ about a remote pivot point, resulting in more than $300 \mu \mathrm{m}$ of total mirror motion.

The actuator design incorporates a unique mechanicallybalanced approach. Many other electrostatic rotary sensors and actuators have been symmetric about their center of rotation $[8,9]$ and thus have been inherently balanced. From an optical design standpoint it would have been difficult to assemble a practical laser system with a symmetric actuator rotating about the desired mirror pivot point, so an alternative approach was taken to design an actuator which rotates a mirror about the desired pivot point but also includes features to mechanically balance the device.

A virtual pivot can be made by attaching two adjacent ends of a pair of flexural beams to the moveable frame and anchoring the other two ends to the substrate [10]. If the beams cross in an Xshape, then for small deflections the center of rotation of the flexure is at the center of the $X$ [11]. If the beams are arranged in a V-shape or do not physically cross, then for small deflections the center of rotation is at the base of the $\mathrm{V}$ or at the intersection of lines passing through the beams.

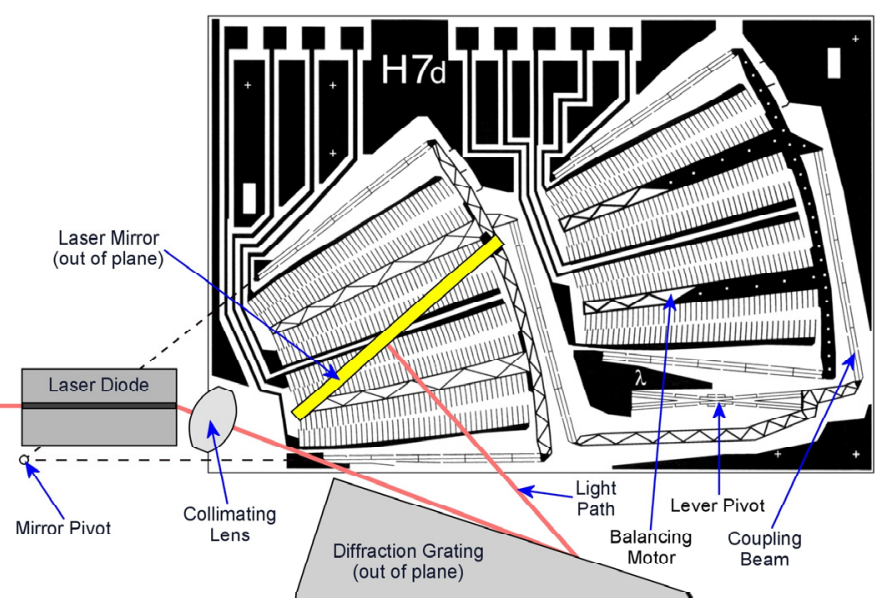

Figure 3. Plan view of laser mirror actuator, laser die, collimating lens and diffraction grating. The actuator chip is 4.3 $\mathrm{mm} \times 3.0 \mathrm{~mm}$. The laser mirror is driven, e.g. clockwise, by its motor while the balancing motor is driven counter-clockwise. The motion is constrained by the pivoting lever and the associated coupling beams to minimize response to external vibration.

To make a laser mirror rotator, two similar rotary actuators with virtual pivots are coupled via a lever with an X-type flexural pivot and driven in a push-pull configuration. A photograph of a mirror motor chip is shown in Fig. 4. The mass of the right-hand motor is designed to be nearly identical to the mass of the left-hand motor and mirror. External accelerations in the plane of the actuator then cause equal and opposite torques on the pivoting lever, eliminating any significant rotation of that lever or the motors it connects. It is feasible to realize a $600 \times$ reduction in mirror rotation due to in-plane acceleration compared to a similar single motor and mirror without this balancing arrangement. Any residual motion of the part is due to slight imbalances between the right-hand and left-hand masses and flexing of the lever due the imposed torques. The response to out-of-plane accelerations is 
reduced by the large out-of-plane stiffness provided by the high aspect ratio of the suspension. This mechanical-balancing concept can also be applied to linear actuators by arranging a similar pivoting lever between two linear actuators of equal mass.

The suspension beams, coupling beams, and flexural pivot include protection fins to improve the sidewall profile during etching [6,7]. These protection features are attached locally on or near the structures, for example, along the length of a suspension beam. Thus while the narrow suspension beams are being etched, ions tend to be channeled in the etch direction, and stray ions from adjacent open areas are blocked by the fins.

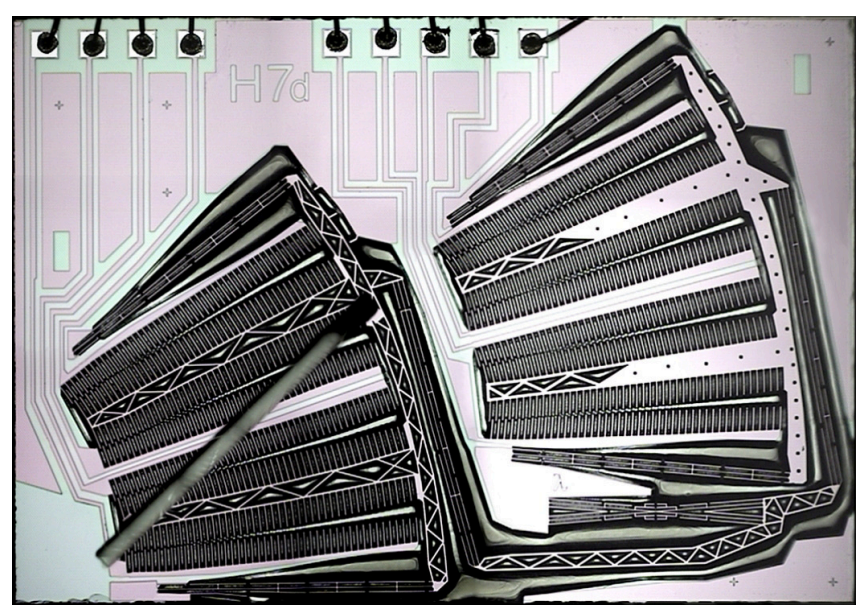

Figure 4. Photograph of laser mirror actuator die after the externally-fabricated mirror is attached. Protection fins are fabricated on the suspension and connector beams to control the sidewall etch profile.

\section{ACTUATOR RESPONSE}

For a closed-loop system it is important to design the actuator to have a transfer function that is monotonic, and have as linear a response as possible. A conventional comb-drive actuator provides a force that is nearly independent of position as long as the combs are interdigitated, but that force is quadratic in applied voltage. For a given maximum drive voltage, the actuator range can be doubled by providing combs that can either "push" or "pull", however the drive circuitry must be designed so that there is no dead-zone or hysteresis in the center of the range. Differential drive can be employed with fully interdigitated combs to linearize the response [8], but for this actuator, that would result in combs more than $400 \mu \mathrm{m}$ long. To resist the side electrostatic forces imposed on them, such long combs would need to be quite wide. However, as the width of the combs is increased to make them stiffer, the motor mass and the comb pitch are increased, reducing the resonant frequency and electrostatic force. Instead, the lengths of the individual combs have been adjusted to substantially linearize the response over the operating range while limiting the maximum comb length to $125 \mu \mathrm{m}$. A graph of rotation vs. drive voltage is shown in Fig. 5 for a typical actuator with a resonant frequency of about $300 \mathrm{~Hz}$.

With a transfer function similar to that in Figure 5, the laser system, including a wavelength-locking servo, can maintain wavelength accuracy of better than $10 \mathrm{pm}(1.25 \mathrm{GHz})$ with an applied acceleration of $5 \mathrm{~g}$ at $50 \mathrm{~Hz}$.

The reliability of the actuators and laser is critically important in a telecommunications application, where a 20 year lifetime is required. The use of purely flexural suspension elements insures that there are no friction or stiction issues during operation. A set of actuators has been collectively cycled over 60 billion times with no measurable change in performance.

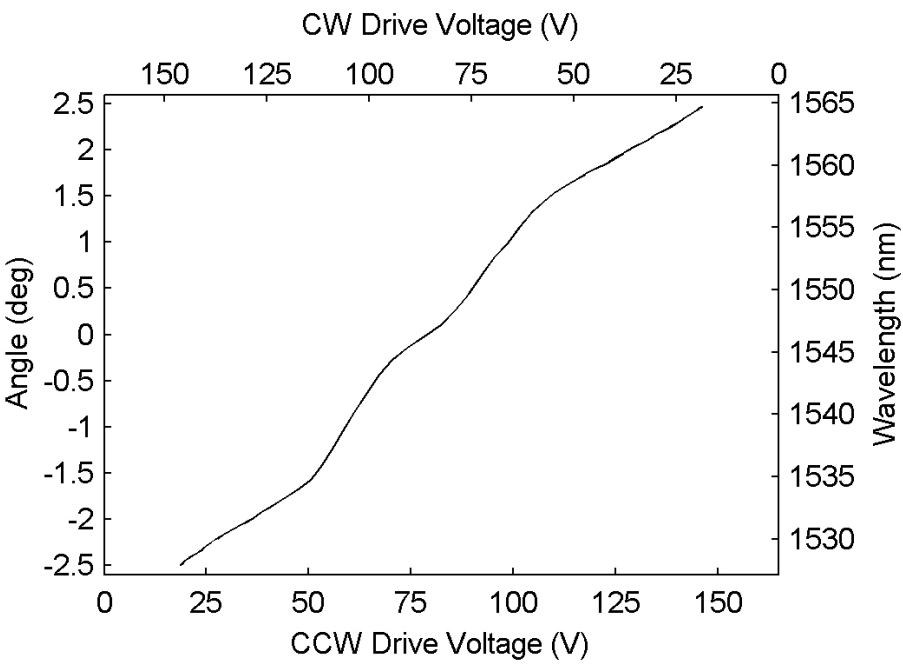

Figure 5. Angular response of the mirror actuator vs. differential drive voltage. The response has been linearized by adjusting the lengths of the individual combs.

\section{LASER PERFORMANCE}

The laser can be tuned and locked on $25 \mathrm{GHz}$ ITU channels over a wavelength range of $38 \mathrm{~nm}$ with greater than $20 \mathrm{~mW}(+13$ $\mathrm{dBm}$ ) of single-mode, fiber-coupled output power, and it has supported transmission of data at $40 \mathrm{~Gb} / \mathrm{s}$ over distances exceeding $800 \mathrm{~km}$ and $10 \mathrm{~Gb} / \mathrm{s}$ over distances exceeding $3000 \mathrm{~km}$. The laser can be tuned to another channel and locked in less than $15 \mathrm{~ms}$. A plot of 100 superimposed optical spectra showing the laser locked to sequential, $25 \mathrm{GHz}$-spaced channels is shown in Fig. 6.

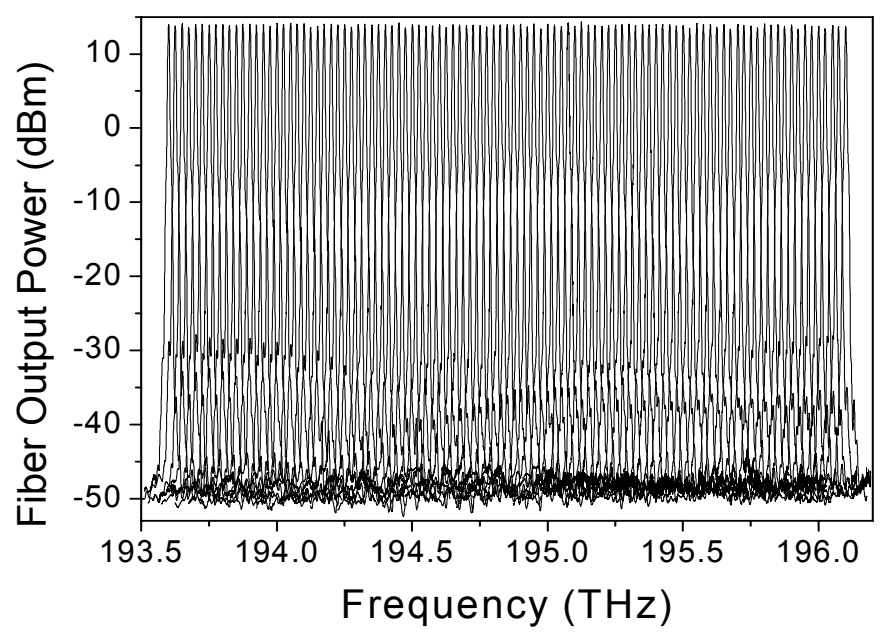

Figure 6. Superimposed optical spectra of laser locked to 100 consecutive $25 \mathrm{GHz}$ ITU optical channels. Fiber-coupled output power is $>20 \mathrm{~mW}(+13 \mathrm{dBm})$ and side mode suppression is $>50$ $d B$. These optical signals have been used to transmit data at 10 $\mathrm{Gb} / \mathrm{s}$ over $3000 \mathrm{~km}$.

With appropriate optical components, similar performance can be obtained in both the C-band (1529-1561 nm) and L-band 
(1566-1607 nm) and with channels spaced by 25,50 or $100 \mathrm{GHz}$. The optical performance of the laser is improved by having a relatively long, external cavity and a highly dispersive diffraction grating. The laser has relative intensity noise (RIN) of less than $-145 \mathrm{~dB} / \mathrm{Hz}$ from $10 \mathrm{MHz}$ to $10 \mathrm{GHz}$, polarization extinction ratio better than $20 \mathrm{~dB}$, and spontaneous emission background more than $50 \mathrm{dBc} / \mathrm{nm}$ below the laser peak value. The fundamental, spontaneous-emission-induced linewidth is less than $1 \mathrm{MHz}$, narrower than that of a typical DFB laser. Such superior optical performance is required to support high data rates over long distances in an optical link.

Very small mechanical fluctuations of the mirror on the actuator in the range of $5-50 \mathrm{kHz}$ kilohertz broaden the observed time-averaged optical linewidth, and a homodyne measurement with a $25 \mu$ s delay gives a time-averaged $3 \mathrm{~dB}$ linewidth of about 2 $\mathrm{MHz}$. This represents an average displacement of the mirror over the $25 \mu$ s measurement interval of about $0.12 \mathrm{~nm}$.

\section{PERFORMANCE UNDER VIBRATION}

As described above, the mirror rotator consists of two identical counter-rotating actuators, coupled in a counterweighted configuration so that the net moment of the device is zero. The balanced actuator design is important for minimizing excitation of the fundamental resonant mode. The residual low-frequency vibrational sensitivity is suppressed with servo control of the actuator. At higher frequencies, conventional viscous damping tends to minimize the coupling between external vibrations and the laser, however some modes are damped more than others. Fig. 7 shows the transfer function of case acceleration to laser frequency variation, as the vibration frequency is swept from $10 \mathrm{~Hz}$ to 10 $\mathrm{kHz}$. The upper graph shows the sensitivity without the wavelength locker servo activated. The lower graph shows the effect of the wavelength locker servo, reducing the laser frequency sensitivity to $15 \mathrm{MHz}$ per applied $\mathrm{G}$ of case acceleration at $10 \mathrm{~Hz}$ vibration frequency.

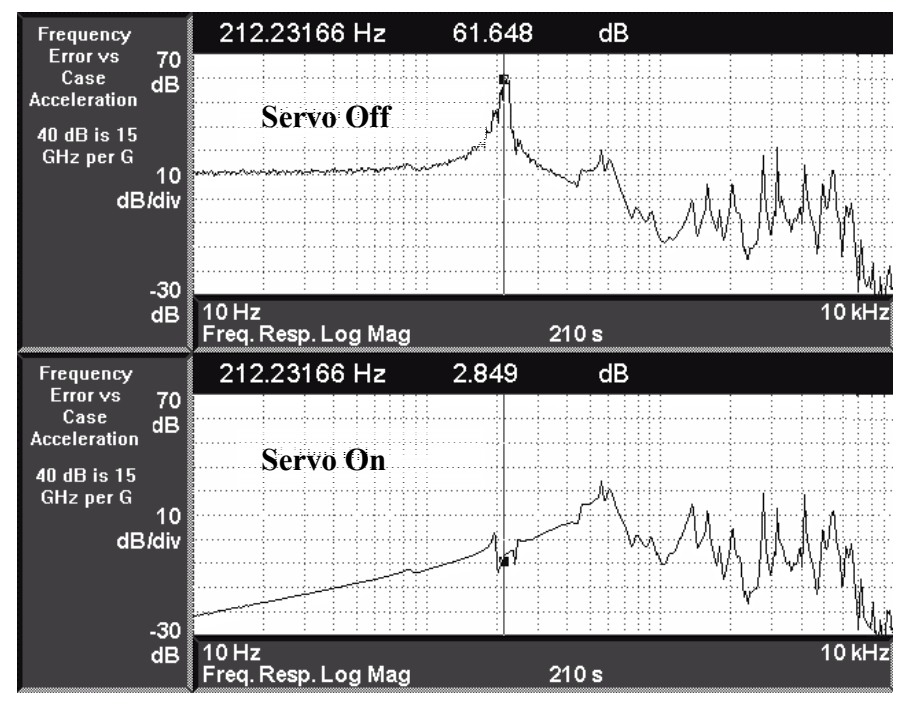

Figure 7. The transfer function in $\mathrm{MHz} / \mathrm{G}$ as the laser is subjected to vibrational excitation from $10 \mathrm{~Hz}$ to $10 \mathrm{kHz}$. The fundamental in-plane response of this actuator at about $212 \mathrm{~Hz}$ has been reduced by about $35 \mathrm{~dB}$ by the servo system.

\section{CONCLUSIONS}

Silicon DRIE microactuators have enabled a small form factor, tunable laser source ideal for many data transmission applications. The performance of the laser meets the optical power, side mode suppression, polarization extinction ratio, relative intensity noise, and linewidth requirements for transmitting $10 \mathrm{~Gb} / \mathrm{s}$ over distances greater than $3000 \mathrm{~km}$. Frequency accuracy of $\pm 1.25 \mathrm{GHz}$ is obtained with closed loop control of the actuator drive voltage. Substantial immunity of the laser system to vibrations is achieved through a combination of mechanical design and active servo control.

\section{ACKNOWLEDGEMENTS}

The authors would like to thank the iolon development team for their help in preparing this paper, including Kathy Jackson for preparing and testing the actuator devices, Jill Berger and Jeff Hutchins for providing the optical data and Al Fennema and Ken Cheung for the servo response data.

\section{REFERENCES}

1. M. G. Littman and H. J. Metcalf, "Spectrally narrow pulsed dye laser without beam expander", Applied Optics, Vol. 17 No. 14, (1978) 2224-2227.

2. K. Liu and M.G. Littman, "Novel geometry for single-mode scanning of tunable lasers", Optics Letters, Vol. 6 No. 3, (1981) 117-118.

3. D. Vakhshoori, P. Tayebati, Chih-Cheng Lu, M. Azimi, P. Wang, Jiang-Huai Zhou and E. Canoglu, "2 $\mathrm{mW} \mathrm{CW}$ single mode operation of a tunable $1550 \mathrm{~nm}$ vertical cavity surface emitting laser with $50 \mathrm{~nm}$ tuning range," Eletronic. Let., 35, 1-2 (1999).

4. F. Sugihwo, M.C. Larson, and J.D. Harris, "Micromachined widely tunable vertical cavity laser diodes", J. Microelectromechanical Systems, Vol. 7, No. 1. (1998) pp. 48-55.

5. X.M. Zhang, A.Q. Liu, V.M. Murukesham, and F.A. Chollet, "Integrated micromachined tunable lasers for all-optical network applications, Proceedings Transducers 2001, June 2001, pp. 13141317.

6. J.H. Jerman, J.D. Grade, J.D. Berger, and J.F. Heanue, "Tunable laser with microactuator", International Publication number WO 01/43241, WIPO, June 2001.

7. J.D. Grade, H. Jerman, and T.W. Kenny, "A large-deflection electrostatic actuator for optical switching applications," Technical Digest of the 2000 Solid State Sensor and Actuator Workshop, Hilton Head, SC, June 2000, pp. 97-100.

8. L.S. Fan, S.J. Woodman, R.C. Moore, L. Crawford, T.C. Reiley, and M.A. Moser, "Batch-Fabricated Area-Efficient MilliActuators," Technical Digest of the 1994 Solid State Sensor and Actuator Workshop, Hilton Head, SC, June 1994, pp 38-42.

9. D.A. Horsley, A. Singh, A.P. Pisano, and R. Horowitz, "Angular Micropositioner for Disk Drives", Proceedings of the Tenth International Workshop on Micro Electro Mechanical Systems, 1997, pp 454-458.

10. J.D. Grade and J.H. Jerman, "MEMS electrostatic actuators for optical switching applications", Technical Digest, Optical Fiber Communication Conference, March 2001, paper WX2-2.

11. S.T. Smith and D.G. Chetwynd, Foundations of Ultraprecision Mechanism Design, Gordon and Beach Science Pub., Amsterdam, 1992, p. 119. 